CLINICAL STUDY

\title{
Effects of the long-acting somatostatin analogue Lanreotide Autogel on glucose tolerance and insulin resistance in acromegaly
}

\author{
B Steffin, B Gutt ${ }^{1}$, M Bidlingmaier, C Dieterle, F Oltmann and J Schopohl \\ Medizinische Klinik Innenstadt, Department of Endocrinology, University of Munich, Munich, Germany and ${ }^{1}$ Department of Endocrinology, Academic \\ Hospital Bogenhausen, Technical University of Munich, Munich, Germany
}

(Correspondence should be addressed to B Steffin; Email: birgit.steffin@med.uni-muenchen.de)

B Steffin and B Gutt contributed equally to this work

\begin{abstract}
Object: Treatment with somatostatin analogues (SA) not only inhibits GH secretion but may also impair insulin secretion. In order to evaluate the influence of SA on glucose metabolism, we investigated insulin resistance (IR) and $\beta$-cell function, using the recommended combination of homeostatic model assessment of IR (HOMA-IR) and $\beta$-cell function (HOMA- $\beta$ ).

Design and methods: This is a prospective, cross-sectional study. We measured fasting insulin, blood glucose and IGF-I. Insulin and blood glucose measurements were taken $120 \mathrm{~min}$ after an oral glucose tolerance test with $75 \mathrm{~g}$ glucose. We studied 51 patients $(27$ female/24 male, age 54 years (20-75)). Eighteen patients were on Lanreotide Autogel (LA) treatment, 33 had no medical treatment. GH-levels of more than $2.5 \mathrm{ng} / \mathrm{ml}$ was reached by $59 \%$ of the patients, $74.5 \%$ had normal IGF-I levels.

Results: We found no significant influence of disease activity on HOMA-IR and HOMA- $\beta$. In the 33 of 51 subjects without any drug treatment, median HOMA- $\beta$ was $170.4 \%$ (36.0-624.0\%). In contrast, in the 18 patients on LA treatment, median HOMA- $\beta$ was found to be significantly lower $(84.2 \%(36.5-346.2 \%)$; $P=0.001)$. Despite this, there was no difference in HOMA-IR in both groups $(2.4(0.7-8.4)$ vs $2.3(0.7-6.1) ; P<0.001)$ despite similar insulin values.

Conclusion: In conclusion, we found that LA decreases $\beta$-cell function significantly without affecting IR. Therefore, we think that insulin secretagogues are probably more effective in the treatment of diabetes mellitus in acromegalic patients on LA therapy than insulin sensitizers.
\end{abstract}

European Journal of Endocrinology 155 73-78

\section{Introduction}

Impaired glucose tolerance (IGT) and type 2 diabetes mellitus (DM) are severe complications frequently affecting acromegalic subjects. Overt diabetes is reported in $19-56 \%$ and IGT in $16-46 \%$ of acromegalic patients (1). Diabetes is most frequent in patients with higher growth hormone $(\mathrm{GH})$ levels and plasma insulin-like growth factor-I (IGF-I) levels show a close correlation with fasting and postprandial glucose (2). Insulin resistance (IR) is reported to be common in acromegaly and is suggested to contribute to abnormal glucose tolerance in acromegalic patients. The mechanism underlying this IR is unclear. In fact, some studies have reported a direct correlation between circulating $\mathrm{GH}$ plasma levels and the degree of glucose intolerance (3). Clinically, diabetes in acromegaly usually resembles type 2. Interestingly, a recent study suggested that the abnormalities of glucose metabolism occurring in acromegaly could contribute to the development of hypertension, which affects over $50 \%$ of these patients. The increased cardiovascular morbidity and mortality associated with acromegaly is, at least in part, a consequence of the increased IR that frequently accompanies GH hypersecretion (4). Hyperglycaemia may worsen in patients treated with somatostatin analogues (SA), because insulin secretion, i.e. $\beta$-cell function, is also suppressed. SAs achieve 'safe' GH and IGF-I concentrations in approximately $60 \%$ of patients, but their inhibitory effect on pancreatic insulin secretion implicates that their overall effect on glucose tolerance is complex and unpredictable (5). The aim of the present study was to elucidate whether acromegalic patients have elevated IR, reduced $\beta$-cell function and whether therapy with the SA Lanreotide Autogel (LA) (Group Ipsen, Paris, France) impairs glucose tolerance or not. 


\section{Patients and methods}

\section{Patients}

In this prospective, cross-sectional study, all patients with acromegaly on lanreotide treatment and without medication, who were seen in our outpatients' clinic in the last 3 years (Department of Endocrinology, Ludwig-Maximilian's University, Munich, Germany), were contacted by mail. Fifty-one patients $(24$ males and 27 females, aged 20-75 years (median 52)) gave informed consent to participate and were all included in this study. The mean period of disease activity was 19 years (1-42 years). This was assessed by patient interview and the onset of acral enlargement to date. Using strict criteria of cure (normalized IGF-I and suppressed GH $<1 \mu \mathrm{g} / \mathrm{dl}$ after oral glucose tolerance test (OGTT)), 17 of our 51 investigated patients were cured by transsphenoidal surgery. Two of the four patients who had additional external irradiation were cured and two were not. Eighteen patients had concomitant medication with the somatostatin analogue LA; nine patients received lanreotide $60 \mathrm{mg} / \mathrm{month}$ and nine, $120 \mathrm{mg} / \mathrm{month}$ for at least 1 year via s.c. administration. Among the 21 active acromegalic patients, 12 are active despite LA and surgery. Fifty-nine percent of the patients reached 'Safe' GH levels $(<2.5 \mathrm{ng} / \mathrm{ml})$ and $74.5 \%$ had normal age- and sex-adjusted IGF-I levels (Table 1). According to WHO criteria, our cohort contained five patients with DM, nine with IGT and 37 with normal glucose tolerance (NGT) (6).

Table 1 Clinical features and treatment characteristics of 51 patients with acromegaly (IGF-I is given as a multiple of upper limit for age normal, $(\times \cup L N))$.

\begin{tabular}{lc}
\hline No. of patients & 51 \\
Sex (M/F) & $24 / 27$ \\
Age (years; median) & 52 \\
Range & $20-75$ \\
Follow-up time & 19 \\
Disease activity (years; median) & $1-42$ \\
Range & \\
Pretreatment (no. of pat.) & 17 \\
Successful surgery & 34 \\
Unsuccessful pituitary surgery & $4 / 34$ \\
Conventional irradiation & $18 / 34$ \\
Lanreotide Autogel treatment & \\
$\quad \times$ ULN of IGF-I in all patients & 0.68 \\
Median & $0.15-2.27$ \\
Range & \\
BMI in all patients & 28.28 \\
Median & $20.48-45.14$ \\
Range & 31 \\
Pituitary deficiency (no. of pat.) & $29 / 31$ \\
Gonadal axis & $24 / 31$ \\
Thyroidal axis & $18 / 31$ \\
Adrenal axis & \\
\hline
\end{tabular}

\section{Methods}

Diabetic status, such as IGT, impaired fasting glucose or DM, was estimated by the OGTT (according to the World Health Organization criteria with $75 \mathrm{~g}$ glucose) (6). Plasma glucose, insulin and serum GH levels were measured every half an hour; IGF-I was measured only once together with fasting glucose. We determined IR (HOMA-IR) and $\beta$-cell function (HOMA- $\beta$ ) by homeostatic model assessment (HOMA). This is a computer model for predicting IR (percentage $\mathrm{R}$ ) and $\beta$-cell deficiency (percentage $\beta)$ (7). Increasing HOMA-IR values indicate increasing IR. Assuming that normal-weight normal subjects aged above 35 years have a $\beta$-cell function of $100 \%$ and an IR (HOMA-IR) of 1, the values for a patient can be assessed from the insulin and glucose concentrations by the formulae (7)

HOMA-IR $=($ insulin $(\mathrm{mU} / \mathrm{l}) \times$ glucose $(\mathrm{mmol} / \mathrm{l})) / 22.5$

HOMA- $\beta(\%)=(20 \times$ insulin $(\mathrm{mU} / \mathrm{l})) /($ glucose

$$
(\mathrm{mmol} / \mathrm{l})-3.5)
$$

\section{Laboratory values}

Serum concentrations of GH were measured using the automated Advantage chemiluminescent assay system (Nichols Diagnostics Institute, Bad Vilbel, Germany). This sandwich-type immunoassay involves a monoclonal capture and a polyclonal detection antibody. Within-assay coefficient of variance (cv) was 3.5, 2.2 and $2.9 \%$ at concentrations $1.4,10.5$ and $28.0 \mu \mathrm{g} / \mathrm{l}$. Between-assay variability at the same concentrations was $7.9,2.7$ and $5.9 \%$. The lower limit of quantification was $0.2 \mu \mathrm{g} / \mathrm{l}$; the linear working range was $0.2-50 \mu \mathrm{g} / \mathrm{l}$. IGF-I levels were measured by the automated Advantage chemiluminescent IGF-I assay system (Nichols Diagnostics Institute). For this method, samples are acidified to separate IGF-I from its binding protein IGFBP3. To prevent re-aggregation of IGF-I and IGFBP 3 and thus to exclude interference of IGFBP 3 , the acidified samples were incubated with excess of IGF-II. The intraassay $\mathrm{CV}$ was $11.5,5.1$ and $3.5 \%$ at concentrations of 42,262 and $522 \mu \mathrm{g} / \mathrm{l}$. At the same concentration, between-assay $\mathrm{Cv}$ was $10.6,10.6$ and $10.2 \%$. The lower limit of quantification was $17 \mu \mathrm{g} / \mathrm{l}$; the linear range was $17-1000 \mu \mathrm{g} / \mathrm{l}$. In this manuscript, all IGF-I values are indicated as a multiple of the upper limit of normal $(\times \mathrm{ULN})$ as calculated using the following formula

$$
\begin{aligned}
\times \mathrm{ULN}= & \text { IGF-I value/95th percentile of age- and } \\
& \text { sex-adjusted normal range. }
\end{aligned}
$$

Insulin levels were measured by the insulin RIA manufactured by Adaltis Italia (S.p.A; Casalecchio di Reno, Italy). Glucose levels were measured by an automated glucose analyser; HbAlc levels were 
determined by HPLC. We used the mean value of fasting measurements for calculating HOMA-IR and HOMA- $\beta$.

\section{Statistical analysis}

StatView (for Microsoft Windows, SAS Campus Drive, Cary, NC, USA) was used for data analysis. For all investigated parameters, group values are expressed as median (and range), unless, otherwise indicated. Statistical significance in the outcome differences was evaluated using nonparametrical statistics (KruskalWallis test) followed by Mann-Whitney $U$ test. Categorical data were analysed with Fisher's test, when appropriate. A $P$-value $<0.05$ was considered as the nominal level of significance, a $P$-value $<0.01$ as the nominal level of high significance.

\section{Results}

\section{Influence of disease activity on IR (HOMA-IR)}

We investigated the influence of disease activity on IR in 51 patients. In the 21 acromegalic patients with active disease, the median HOMA-IR was $2.3(0.7-8.4)$. There was no significant difference to the 30/51 cured patients $(2.4(0.7-6.1))$. To exclude the influence of lanreotide, we separately investigated only those 33 patients who were off medical treatment. Even in this subgroup, there was no significant difference in HOMAIR between the 24/33 normalized and the 9/33 active patients, 2.5 (0.7-6.1) and $2.1(1.1-3.3)$ respectively.

\section{Influence of lanreotide treatment on IR (HOMA-IR)}

Furthermore, to investigate the influence of LA on IR, we compared HOMA-IR in all patients treated with lanreotide to those who did not have any medical treatment. In the $18 / 51$ patients who were on lanreotide, the median HOMA-IR was $2.4(0.7-8.4)$. There was no statistical difference in IR to those 33/51 without lanreotide treatment (2.3, range 0.7-6.1; $P=$ NS (Fig. 1)). To exclude the possibly existing effect of disease activity, we performed subgroup analysis. We investigated the influence of medication on HOMA-IR in patients with 'active disease' $(21 / 51)$ and 'controlled disease' (30/51) separately. Neither in active nor in controlled patients, an influence of lanreotide on HOMA-IR could be shown (Table 2). Comparing HOMA-IR separately in patients suffering from frank diabetes and those with IGT and NGT, we found that diabetic patients and IGT patients had higher HOMA-IR (median $3.13(1.47-8.37)$ ) than those who did not suffer from DM (median $2.23(0.68-5.57) ; P=0.02)$. DM patients and IGT patients did not differ significantly in HOMA-IR $(P=\mathrm{NS})$.

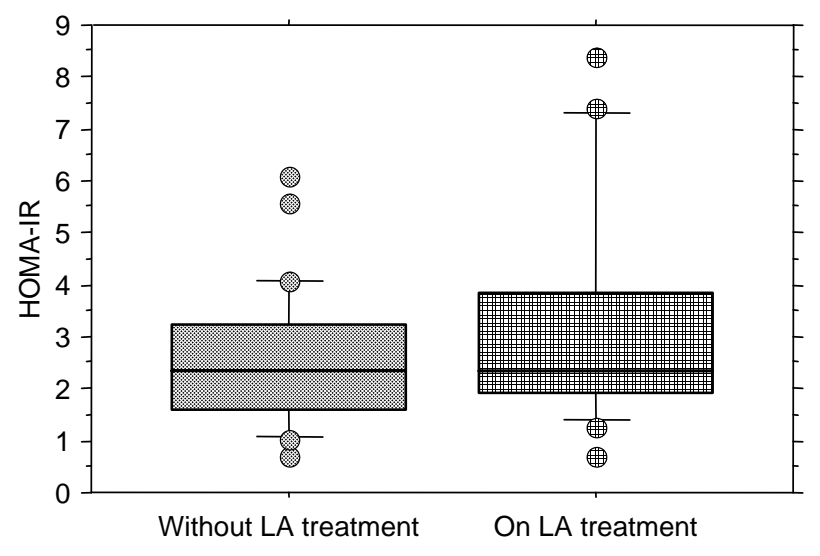

Figure 1 Comparison of HOMA-IR as parameter of insulin resistance in acromegalic patients on treatment with Lanreotide Autogel (LA; $n=18)$ to those without medical treatment $(n=33)$ shows no significant influence of LA treatment on patients' IR $(P=\mathrm{NS})$. (Box plot: 10th, 25th, 50th (median), 75th, 90th percentiles, values $>90$ th and $<10$ th percentile as points).

\section{Influence of disease activity on $\beta$-cell function (НОMA- $\beta)$}

We investigated the influence of disease activity on $\beta$-cell function. In the 21 of 51 patients with active disease, the median HOMA- $\beta$ was $136.8 \%$ (range $37.2-$ $346.2 \%)$. There was no significant difference to the $30 / 51$ normalized patients (156.5\% (36.0-624.0\%)). Reanalysing only the 33 patients who did not receive LA therapy showed no significant difference in HOMA- $\beta$ between the $23 / 33$ cured and the $9 / 33$ active patients, $177.1 \%(36.0-624.0 \%)$ and $149.1 \%(46.9-270.0 \%)$ respectively.

\section{Influence of lanreotide treatment on $\beta$-cell function (HOMA- $\beta$ )}

To investigate the influence of lanreotide treatment on $\beta$-cell function, we analysed HOMA- $\beta$ in all the 51 patients. We compared those $18 / 51$ patients treated by lanreotide to the $33 / 51$ patients who did not have medical treatment. In those $33 / 51$ patients without LA treatment, the median HOMA- $\beta$ was $170.4 \%$ (36.0-624.0\%). There was a significantly decreased $\beta$-cell function in the 18/51 patients who were on lanreotide treatment with a median HOMA- $\beta$ of $84.2 \%$ (36.5-346.2\%; $P=0.001$ (Fig. 2 )). To exclude the influence of disease activity, we analysed subgroups. In patients with active disease on LA $(n=12)$, $\beta$-cell function was significantly reduced (median HOMA$\beta 91.2(37.2-270.0)$ ) as against nine active patients without LA (149.1 (46.9-346.2); $P=0.05)$. In patients with controlled disease, we found in those six patients on LA treatment, a significantly lower HOMA- $\beta$ (74.4 (36.5136.2)) than in those 24 patients without LA treatment (177.1 (36.0-624.0); $P=0.002$; Table 2). In IGT and DM patients $(n=14)$ there is no difference in HOMA- $\beta$ between those who are on LA treatment and those who 
Table 2 Parameters of glucose metabolism; comparison of patients with active acromegaly (AM) to normalized patients and patients on treatment with LA to those without medical treatment and patients with normal glucose tolerance (NGT) to those with impaired glucose tolerance or diabetes mellitus (IGT/DM).

\begin{tabular}{|c|c|c|c|c|c|c|}
\hline & $\begin{array}{c}\text { Active AM on LA } \\
\text { treatment }\end{array}$ & $\begin{array}{l}\text { Active AM without } \\
\text { LA treatment }\end{array}$ & $\boldsymbol{P}$ & $\begin{array}{l}\text { Controlled AM on } \\
\text { LA treatment }\end{array}$ & $\begin{array}{c}\text { Controlled AM } \\
\text { without LA } \\
\text { treatment }\end{array}$ & $\boldsymbol{P}$ \\
\hline$n$ & 12 & 9 & & 6 & 24 & \\
\hline $\mathrm{FBG}(\mathrm{mmol} / \mathrm{l})$ & 5.89 (4.39-9.78) & $4.89(4.28-5.89)$ & 0.004 & $5.64(5.33-7.33)$ & $4.81(3.83-5.72)$ & 0.018 \\
\hline $\mathrm{FI}(\mathrm{mU} / \mathrm{l})$ & $10(3-30)$ & $10(6-25)$ & NS & $8.5(5-14)$ & $12(3-28)$ & NS \\
\hline HOMA-IR & $2.51(0.68-0.84)$ & $2.06(1.10-3.33)$ & NS & $2.30(1.26-3.46)$ & $2.47(0.69-6.08)$ & NS \\
\hline HOMA- $\beta$ & $91.2(37.2-270.0)$ & $149.1(46.9-346.2)$ & 0.05 & 74.4(36.5-136.2) & $177.1(36.0-624.0)$ & 0.002 \\
\hline BG120 (mmol/l) & $7.14(4.78-16.61)$ & $5.00(2.83-16.61)$ & 0.036 & $5.42(4.39-9.61)$ & $5.42(3.56-9.83)$ & NS \\
\hline Ins.120 (mU/l) & 78 (15-247) & $33(10-130)$ & NS & $44.5(21-303)$ & $68.5(13-290)$ & NS \\
\hline
\end{tabular}

FBG, fasting blood glucose; FI, fasting Insulin; BG120, Ins120, blood glucose and insulin 120 min after oral glucose load.

are not. Those 37 NGT patients who are under medical therapy with LA $(n=10)$ have a significantly reduced $\beta$-cell function compared to NGT patients who are not on medical therapy $(n=27 ; P<0.006)$.

\section{Blood glucose and insulin values and body mass index (BMI)}

The median fasting insulin value was $10 \mathrm{mU} / \mathrm{l}$ (3.0-30.0 mU/l). Both 'active' and 'controlled' patients showed no significant differences concerning insulin values independently from LA therapy (Fig. 3, Table 2).

The FBG was in median $5.17 \mathrm{mmol} / \mathrm{l}(3.83-$ $9.78 \mathrm{mmol} / \mathrm{l})$. Both in 'active' and 'controlled' patients, FBG was significantly higher in patients on LA treatment $(P=0.004$ and 0.018 respectively; Fig. 3, Table 2). Active patients did not differ in FBG from inactive ones, but we found a significant difference in patients on LA treatment (median FBG was $5.75 \mathrm{mmol} / \mathrm{l}$ (4.61$9.78 \mathrm{mmol} / \mathrm{l})$ ) to those without medication (4.83 mmol/l $(3.83-5.89 \mathrm{mmol} / \mathrm{l}) ; P<0.001)$. There was no difference in distribution of DM/IGT between active and inactive patients without LA treatment. Under LA therapy, the number of DM/IGT rose to a statistically significant relation $(P<0.05)$. Only $18 \%$ of those 33 patients without LA treatment had IGT/DM, whereas $44 \%$ of patients on LA treatment had IGT/DM. There is no significant correlation between BMI and HOMA- $\beta$, but a positive correlation was found between HOMA-IR and BMI $(P=0.007, r=0.432)$. In BMI, there was no difference between patients on LA and those without LA. Patients' mean BMI was $28.18(20.48-45.14 ; n=51)$.

\section{Discussion}

Active acromegaly is frequently discussed to be accompanied by IR and IGT. These factors are assumed to play an important role in the cardiovascular risk of acromegalic patients (8). Considering the studies conducted on this subject reveal that there is no 'standardized' way of estimating glucose metabolism. So far, estimation of glucose metabolism has been mostly performed by measuring $\mathrm{HbAlc}$ and fasting blood glucose. Only few standardized studies in small collectives have been performed to investigate glucose metabolism under treatment with SA. Therefore, effects of SA on glucose metabolism might be underestimated. In this study, we considered the influence of LA treatment on insulin sensitivity and $\beta$-cell function in a larger cohort of acromegalic patients. The estimates of $\beta$-cell function and insulin sensitivity obtained by the HOMA method have been shown to correlate with estimates obtained by the gold standard, hyperglycaemic and euglycaemic clamps respectively (3). HOMA-IR and HOMA- $\beta$ are recommended to be looked at as one entity. Otherwise, it is not possible to distinguish a decrease in HOMA- $\beta$ as a result of a lower insulin secretion from a decrease in IR (9). In our cohort of 51 patients, we found that there was no difference in HOMA-IR between patients on LA treatment and those without LA treatment, and between patients with active and inactive acromegaly, but HOMA-IR was found to be elevated in all patients. In the small investigations in 12 and seven patients on octreotide, hyper- or euglycaemic clamps have been performed so far $(10,11)$. In contrast to our results, Baldelli found that in NGT patients, glucose levels worsened during SA therapy, but not in IGT or DM patients. Both studies found that the

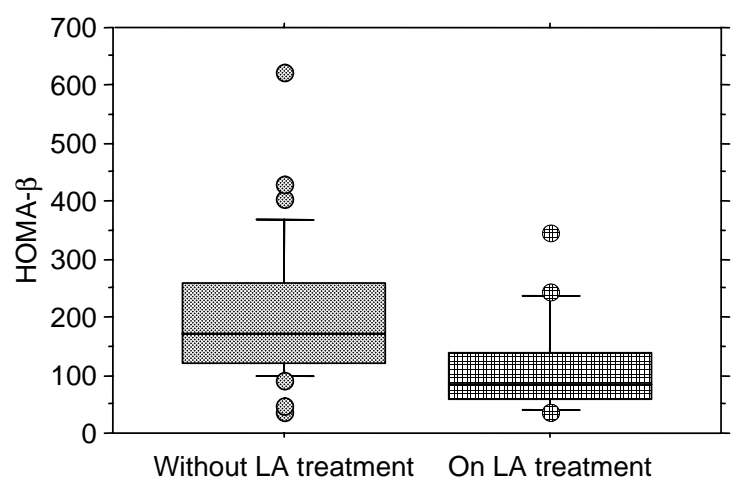

Figure 2 In patients on LA treatment, a significantly lower HOMA- $\beta$ as marker of a suppressed $\beta$-cell function can be found $(P<0.001)$. 
Patients in controlled disease
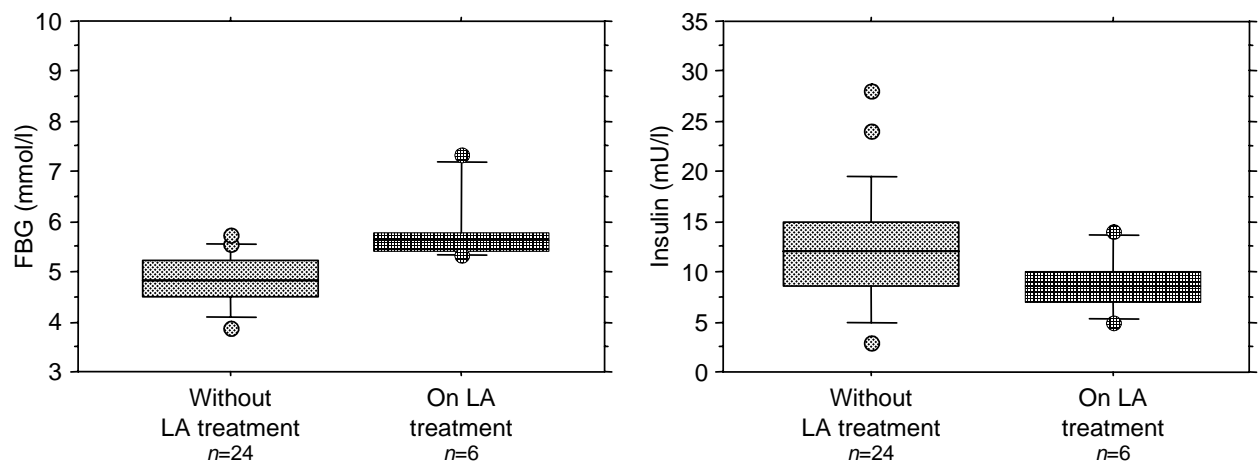

Patients in active disease
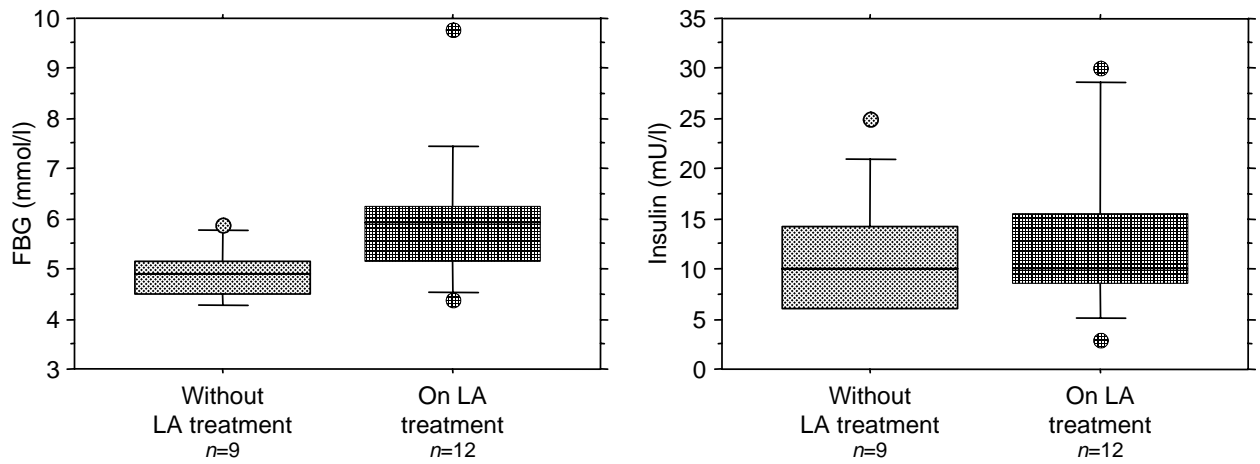

Figure 3 Investigating patients' FBG and insulin values, we found that in both subgroups, patients with 'active' and 'controlled' acromegaly, FBG was significantly higher in patients on LA treatment. There was no significant difference concerning insulin values independently from LA treatment in 'active' and 'controlled' disease.

treatment with the SA octreotide reduces IR with a slight, but significant deterioration of glucose homeostasis in the nondiabetic patients. In 1995, Breidert et al. investigated eight patients' OGTT and euglycaemic clamp results before and after treatment with octreotide $300-600 \mu \mathrm{g} / \mathrm{d}$ with no changes for IR after treatment (12). In our cohort, $\beta$-cell function (HOMA- $\beta$ ) was reduced in patients on LA therapy. Concerning $\beta$-cell function, we found that disease activity does not influence pancreatic secretory function, but medical therapy does (difference between patients on LA treatment and those without any medication). These findings reinforce the hypothesis that the reduced insulin secretion after LA treatment is a direct consequence of a suppressive effect of these drugs. Our findings are contradictory to the findings of Jaffrain-Rea et al. who found a marked decrease in IR after successful surgery in the acromegalic patients (4). The reason for this difference is probably the low acromegalic activity in many of our patients with only slightly elevated GH and IGF-I levels. The decrease in GH needed for cure is too low to have a significant influence on IR. In concordance with our results, investigating the 3-h profiles after s.c. administration of $50 \mu \mathrm{g}$ octreotide in seven acromegalic patients, Verschoor et al. also found that insulin secretion is suppressed; six out of seven patients had slightly higher postprandial glucose values which did not deteriorate further during long-term treatment with octreotide $100 \mu \mathrm{g}$ thrice daily (13). In contrast to the reduced $\beta$-cell function in the HOMA model in our study with LA, Quabbe et al. found in the beginning of octreotide treatment that there was a decrease in the mean 24-h serum insulin concentrations. However, after long-term treatment, an increase in insulin concentrations returned to pretreatment values after 12 months of therapy, whereas glucose levels remained at pathologically elevated levels (14). Newer studies investigated 5, 7 and 152 patients treated with the $\mathrm{GH}$ receptor antagonist pegvisomant $(15,16,17)$. Using only HOMA models, Drake et al. found an unchanged IR in patients converted from stable doses of depot octreotide to pegvisomant with an improvement in $\beta$-cell function (17). In both the other studies, the authors found an improvement in FBG and insulin levels, but IR and $\beta$-cell function were not investigated using the standardized methods like euglycaemic clamp or HOMA models, so unfortunately, no statement on IR or $\beta$-cell function can be made. In terms of previously performed studies, we only found the cross-sectional study of Kasayama et al., who dealt with the evaluation of both HOMA-IR and HOMA- $\beta$ to investigate insulin sensitivity and $\beta$-cell function as 
recommended by Wallace et al. $(3,9)$. The results of the current study support the findings of Wallace et al. (3, 9)- a significantly elevated IR, especially in patients with IGT/DM and reduced HOMA- $\beta$. It therefore appears that reduced $\beta$-cell function, coupled with lower serum insulin levels, indicates a diabetic glucose metabolism with increased IR. However, none of Kasayama's patients had SA treatment. In our cohort, patients with LA treatment in particular, had significantly decreased $\beta$-cell function (HOMA- $\beta$ ), but no difference in IR levels in patients without medical treatment. However, we concede that in our cohort, there is a large proportion of IGT/DM patients in the subgroup of patients on LA treatment. Looking more precisely at their insulin and FBG values, we found that LA-treated patients had significantly higher FBG values at slightly lower (NS) insulin values compared to patients who are not on LA medication, which explains that HOMA-IR as product of insulin and glucose values does not differ significantly, but HOMA- $\beta$ as the quotient of insulin and FBG does. Finally, it remains questionable whether LA treatment itself lowers $\beta$-cell function or if LA medication increases insulin requirements by increasing IR. Our results indicate that an isolated consideration of HOMA-IR or HOMA- $\beta$ is perhaps not appropriate because of the various interactions in regulating glucose homeostasis. It may be more useful to judge both HOMA-IR and HOMA- $\beta$ to estimate the change in one of the components (9).

Preliminary studies on SA investigating IR and $\beta$-cell function have been clamp studies conducted with octreotide on small collectives. Knowing that the computer-solved model by Matthews et al. (7) correlates highly with clamping investigations, we point out by our data that LA also decelerates insulin secretion and thus reduces HOMA- $\beta$. Furthermore, LA-treated patients showed a significantly higher incidence of IGT and DM, whereas IR is not affected. We summarize that for therapy of DM in acromegalic patients on LA treatment, insulin secretagogues are more favourable than insulin sensitizers.

\section{References}

1 Colao A, Ferone D, Marzullo P \& Lombardi G. Systemic complications of acromegaly: epidemiology, pathogenesis, and management. Endocrine Reviews 200425 102-152.

2 Clemmons DR, Van Wyk JJ, Ridgway EC, Kliman B, Kjellberg RN \& Underwood LE. Evaluation of acromegaly by radioimmunoassay of somatomedin-C. New England Journal of Medicine 1979301 1138-1142.

3 Kasayama S, Otsuki M, Takagi M, Saito H, Sumitani S, Kouhara H, Koga M, Saitoh Y, Ohnishi T \& Arita N. Impaired beta-cell function in the presence of reduced insulin sensitivity determines glucose tolerance status in acromegalic patients. Clinical Endocrinology (Oxford) 200052 549-555.

4 Jaffrain-Rea ML, Moroni C, Baldelli R, Battista C, Maffei P, Terzolo M, Correra M, Ghiggi MR, Ferretti E, Angeli A, Sicolo N, Trischitta V, Liuzzi A, Cassone R \& Tamburrano G. Relationship between blood pressure and glucose tolerance in acromegaly. Clinical Endocrinology (Oxford) 200154 189-195.

5 Ronchi C, Epaminonda P, Cappiello V, Beck-Peccoz P \& Arosio M. Effects of two different somatostatin analogs on glucose tolerance in acromegaly. Journal of Endocrinological Investigation $2002 \mathbf{2 5}$ 502-507.

6 World Health Organization definition, diagnosis and classification of diabetes mellitus and its complications. 1999.

7 Matthews DR, Hosker JP, Rudenski AS, Naylor BA, Treacher DF \& Turner RC. Homeostasis model assessment: insulin resistance and beta-cell function from fasting plasma glucose and insulin concentrations in man. Diabetologia 198528 412-419.

8 Colao A, Cuocolo A, Marzullo P, Nicolai E, Ferone D, Della Morte AM, Petretta M, Salvatore M \& Lombardi G. Impact of patient's age and disease duration on cardiac performance in acromegaly: a radionuclide angiography study. Journal of Clinical Endocrinology and Metabolism $1999 \mathbf{8 4} 1518-1523$.

9 Wallace TM, Levy JC \& Matthews DR. Use and abuse of HOMA modeling. Diabetes Care 200427 1487-1495.

10 Baldelli R, Battista C, Leonetti F, Ghiggi MR, Ribaudo MC, Paoloni A, D’Amico E, Ferretti E, Baratta R, Liuzzi A, Trischitta V \& Tamburrano G. Glucose homeostasis in acromegaly: effects of long-acting somatostatin analogues treatment. Clinical Endocrinology (Oxford) $200359492-499$.

11 Ho KK, Jenkins AB, Furler SM, Borkman M \& Chisholm DJ. Impact of octreotide, a long-acting somatostatin analogue, on glucose tolerance and insulin sensitivity in acromegaly. Clinical Endocrinology (Oxford) 199236 271-279.

12 Breidert M, Pinzer T, Wildbrett J, Bornstein SR \& Hanefeld M. Long-term effect of octreotide in acromegaly on insulin resistance. Hormone and Metabolic Research 199527 226-230.

13 Verschoor L, Lamberts SW, Uitterlinden P \& Del Pozo E. Glucose tolerance during long term treatment with a somatostatin analogue. British Medical Journal (Clinical Research Edition) 1986 293 1327-1328.

14 Quabbe HJ \& Plockinger U. Dose-response study and long term effect of the somatostatin analog octreotide in patients with therapy-resistant acromegaly. Journal of Clinical Endocrinology and Metabolism 198968 873-881.

15 van der Lely AJ, Hutson RK, Trainer PJ, Besser GM, Barkan AL, Katznelson L, Klibanski A, Herman-Bonert V, Melmed S, Vance ML, Freda PU, Stewart PM, Friend KE, Clemmons DR, Johannsson G, Stavrou S, Cook DM, Phillips LS, Strasburger CJ, Hackett S, Zib KA, Davis RJ, Scarlett JA \& Thorner MO. Long-term treatment of acromegaly with pegvisomant, a growth hormone receptor antagonist. Lancet $20013581754-1759$.

16 Rose DR \& Clemmons DR. Growth hormone receptor antagonist improves insulin resistance in acromegaly. Growth Hormone and IGF Research 200212 418-424.

17 Drake WM, Rowles SV, Roberts ME, Fode FK, Besser GM, Monson JP \& Trainer PJ. Insulin sensitivity and glucose tolerance improve in patients with acromegaly converted from depot octreotide to pegvisomant. European Journal of Endocrinology $2003 \mathbf{1 4 9} 521-527$.

Received 10 October 2005

Accepted 21 April 2006 